\title{
A Fundamental Study on the High-Power Fiber Laser Cleaning for Removing the Multi-Layer Coating
}

\author{
Jong-Do Kim*, ${ }^{*}$ Ji-Eon Kim**, Moo-Keun Song*** and Jong-Myoung Lee**** \\ *Division of Marine Engineering, Korea Maritime \& Ocean Univ., Busan, 49112, Korea \\ **Ocean Science and Technology School, Korea Maritime \& Ocean Univ., Busan, 49112, Korea \\ ***Laser Advanced Machining Support Center, Korea Maritime \& Ocean Univ., Busan, 49112, Korea \\ ****IMT Co., Ltd., Suwon, 16675, Korea
}

†Corresponding author : jdkim@kmou.ac.kr

(Received August 11, 2020 ; Revised September 7, 2020 ; Accepted September 21, 2020)

\begin{abstract}
The mechanical cleaning technology used in existing coating removal processes, such as blasting and grinding, has various problems, including environmental pollution owing to fine dust, worker welfare problems, and increased process time. As an alternative, an eco-friendly laser cleaning technology has attracted attention. However, research on high-power laser cleaning technology for removing thick coating layers and repairing large areas is limited. In this study, a high-power fiber laser with an average power of $1 \mathrm{~kW}$ was used to conduct cleaning experiments to remove a the multi-layer coating with a thickness of approximately $340 \mu \mathrm{m}$; this coaing is applied to improve the corrosion resistance of steel used for shipbuilding. As a result of experiments based on the main process parameters, such as energy density, average power, and beam mode, the cleaning performance can be significantly improved under a high power density. Moreover, because it exhibits a large difference in surface roughness controllability for each beam mode, it is estimated that the utilization of laser cleaning technology can be maximized depending on the application part.
\end{abstract}

Key Words : High-power laser cleaning process, Eco-friendly technology, Multi-layer coating

\section{Introduction}

A multi-layer coating is a coating system that allows steel structures placed in severe corrosive environments such as marine structures, power plants, and ships to withstand extended exposure to such corrosive conditions. In particular, multi-layer coatings used in the shipbuilding industry consist of multiple layers since a single layer coating is unable to provide excellent adhesive properties, corrosion resistance, and aesthetics ${ }^{1)}$.

Over time, these multi-layer coatings also need to be removed and recoated if they reach the end of their service life or accumulate damage such scratches. Mechanical cleaning technologies such as blasting and grinding have been applied to current coating removal processes, but these techniques suffer from serious environmental pollution, present risks to the health and safety of workers, and face problems of increased lead time due to the hazardous processing environment. To overcome these challenges, there is a growing need for the development of laser cleaning technology employing an environmentally friendly laser heat source for the removal of coating layers ${ }^{2,3}$.

Existing studies on coating layer removal primarily consist of research on the removal of a single layer of epoxy paint and primer according to process parameters using a low-power laser with an average power less than $500 \mathrm{~W}^{4-10}$. Moreover, Marimuthu et al. extended the use of laser cleaning for paint removal to the removal of various contaminants such as TiN coating, oil, and grease on the surface of a material ${ }^{11,12)}$. However, when a low-power laser is used to remove coating layers of thick films that have a thickness of hundreds of micrometers similar to multi-layer coatings as well as oxide layers that have a high boiling point and are more 
tightly bound than coating layers, the cleaning time may significantly increase and complete removal may be difficult. Therefore, it is necessary to develop a cleaning technology employing a high-power laser with an average power above $500 \mathrm{~W}$. Shamsujjoha et al. used a Nd:YAG laser with an average power above $1 \mathrm{~kW}$ to remove paint with a thickness of approximately $200 \mu \mathrm{m}$ and subsequently characterized the mechanical properties and morphology of the cleaned surface, but discussion on the dominant processing parameters during laser cleaning is lacking ${ }^{13,14)}$. Schmidt et al. investigated the effect of average power and energy density during laser cleaning by using a high-power diode laser with an average power of $2.5 \mathrm{~kW}$ to remove a $350 \mu \mathrm{m}$-thick coating layer composed of 5 layers. However, analysis on changes in the surface and cross-section of the cleaned area with respect to processing parameters was not performed ${ }^{15)}$. As described above, high-power laser cleaning is known to be highly advantageous for the removal of coating layers of thick films, but there is still a substantial lack of research on this technology.

Therefore, in this work, high-power fiber laser cleaning at an average power of $1 \mathrm{~kW}$ was employed for the removal of multi-layer coatings in shipbuilding, and the effect of laser cleaning according to energy density and beam mode at each power setting was investigated from various aspects.

\section{Experimental materials and methods}

The material used in experiments is a multi-layer coated steel, and the cross-sectional image of the test specimen is presented in Fig. 1. The coating system consists

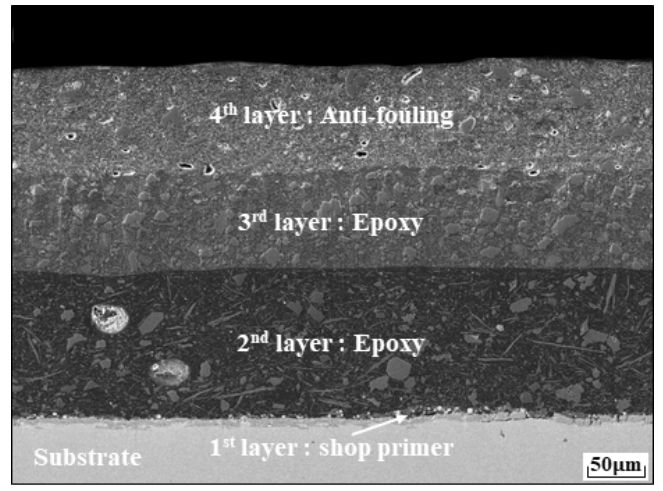

Fig. 1 Cross-section of the specimen of 4 different layers. The first layer is a shop primer with a thickness of approximately $10 \mu \mathrm{m}$. The second and third layers are coated with epoxy paint, and the thickness of each layer is approximately $130 \mu \mathrm{m}$ and $100 \mu \mathrm{m}$, respectively. A red anti-fouling paint is applied at the top layer, and the thickness of the coating layer is approximately $100 \mu \mathrm{m}$, resulting in an overall coating layer thickness of $340 \mu \mathrm{m}$.

Fig. 2 shows the laser cleaning equipment used in experiments, which employs a Q-switching fiber laser with an average power of $1 \mathrm{~kW}$. Specifications of the laser cleaning equipment are presented in Table 1 . The optical system for cleaning consists of a $1.2 \times 1.2 \mathrm{~mm}$ square beam with a multi-mode beam mode, 2D scanner, and scan lens. The laser beam irradiated onto the test specimen was fixed at a pulse overlap rate $\left(R_{\mathrm{po}}\right)$ of $50 \%$ and line overlap rate $\left(R_{\mathrm{lo}}\right)$ of $20 \%$. Detailed experimental conditions are listed in Table 2. Experiments were conducted by varying the energy density $\left(D_{\mathrm{e}}\right)$, which is the main parameter during laser cleaning.

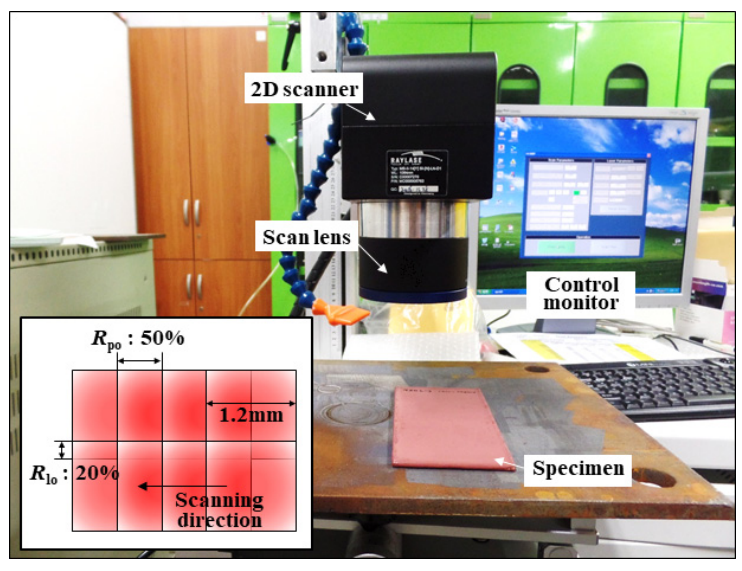

Fig. 2 Setup of experimental equipment for laser cleaning

Table 1 Specification of laser cleaning equipment

\begin{tabular}{|c|c|}
\hline Laser type & Q-switching fiber laser \\
\hline Beam mode & Multi mode \\
\hline Average power $\left(P_{a}\right)$ & $1 \mathrm{~kW}$ \\
\hline Pulse duration time $\left(\tau_{p}\right)$ & $100 \mathrm{~ns}$ \\
\hline Focal spot diameter & $1.2 \times 1.2 \mathrm{~mm}$ \\
\hline Cooling system & Water cooling \\
\hline Maker & IMT \\
\hline
\end{tabular}

Table 2 Experimental conditions with energy density

\begin{tabular}{|c|c|c|c|c|c|c|c|}
\hline $\begin{array}{l}\text { Parameter } \\
D_{\mathrm{e}}\left(\mathrm{J} / \mathrm{cm}^{2}\right)\end{array}$ & $f_{\mathrm{p}}(\mathrm{kHz})$ & $E_{\mathrm{p}}(\mathrm{mJ})$ & $P_{\text {ave }}(\mathrm{W})$ & $v(\mathrm{~m} / \mathrm{s})$ & $R_{\mathrm{po}}(\%)$ & $R_{\mathrm{lo}}(\%)$ & $\tau_{p}(\mathrm{~ns})$ \\
\hline 5.9 & 17 & 58.8 & \multirow{3}{*}{1000} & 8.5 & \multirow{3}{*}{50} & \multirow{3}{*}{20} & \multirow{3}{*}{100} \\
\hline 7.1 & 15 & 71.4 & & 7 & & & \\
\hline 8.3 & 12 & 83.3 & & 6 & & & \\
\hline
\end{tabular}




\section{Results and discussion}

\subsection{Effect of energy density}

The effect of energy density during laser cleaning was investigated by changing the energy density to $5.9 \mathrm{~J} / \mathrm{cm}^{2}$, $7.1 \mathrm{~J} / \mathrm{cm}^{2}$, and $8.3 \mathrm{~J} / \mathrm{cm}^{2}$. Representative images of the surface and cross-section of the laser-cleaned area based on the number of scans at an energy density of $5.9 \mathrm{~J} / \mathrm{cm}^{2}$ are shown in Fig. 3. The red anti-fouling paint at the topmost layer can be observed in the sample before laser cleaning, while the anti-fouling paint is removed after 25 scans and the third layer is exposed. The third layer is removed after 35 scans, and the second layer remains; increasing the number of scans to 45 demonstrates that all coating layers on the surface of the base metal are removed.

X-ray diffraction (XRD) analysis was conducted to examine the change in composition of the laser-cleaned surface according to the number of scans, and the results are shown in Fig. 4. The number of scans was varied from 25 to 45 at intervals of 5, and the change in composition of each layer was clearly distinguishable. Components of the anti-fouling paint were detected in the uncleaned test specimen, while those of epoxy paint were detected after 25 35 scans. In addition, some of the base metal was exposed as more paint layers were removed, resulting in the detection of $\mathrm{Fe}$, the element of the base metal. When the number of scans was increased above 40 , all components of the paint were re-

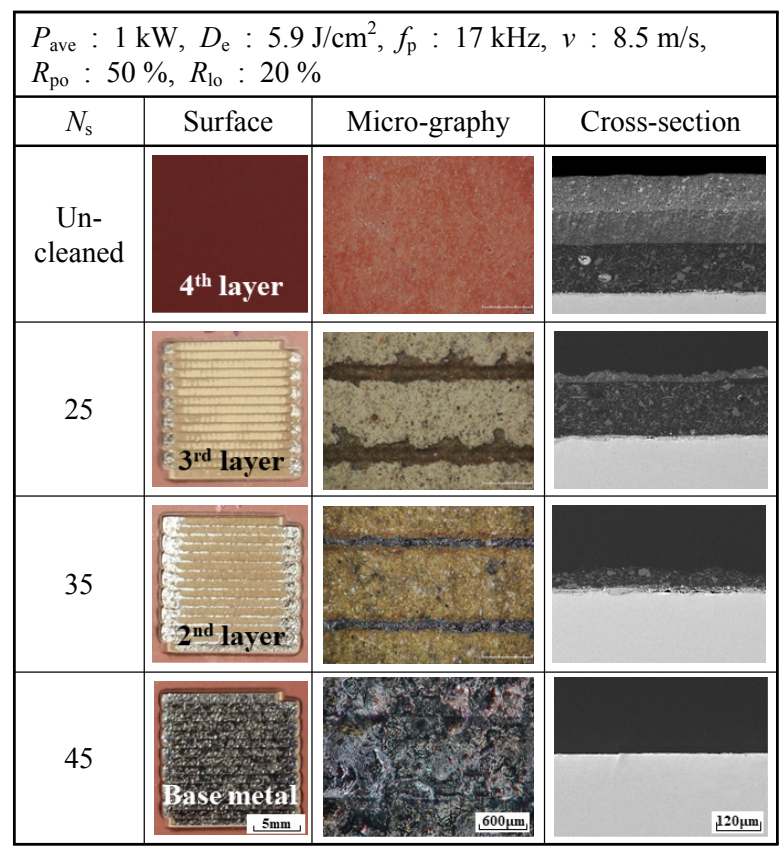

Fig. 3 Surface and cross-section of the laser cleaned surface with the number of scans in De $: 5.9 \mathrm{~J} / \mathrm{cm}^{2}$ moved and only the elements of the base metal were detected. However, comparing the XRD results and surface images suggested that complete removal of all coating layers was achieved at 45 scans.

Fig. 5 shows the change in residual coating thickness with respect to increasing number of scans at each energy density. It can be seen that the thickness gradually decreases with increasing number of scans as the coating layers become vaporized by the laser heat source, and complete removal is achieved with 45 scans at an energy density of $5.9 \mathrm{~J} / \mathrm{cm}^{2}, 40$ scans at $7.1 \mathrm{~J} / \mathrm{cm}^{2}$, and 35 scans at $8.3 \mathrm{~J} / \mathrm{cm}^{2}$. As the energy density increases, the amount of laser energy irradiated on the material increases, making it easier to raise the temperature of the surface to the boiling point of the paint. Thus, at a higher energy density, the relative amount of material that is evaporated and removed upon each laser scan increases, such that removal of the coating layers can be achieved with relatively fewer scans ${ }^{7,10)}$.

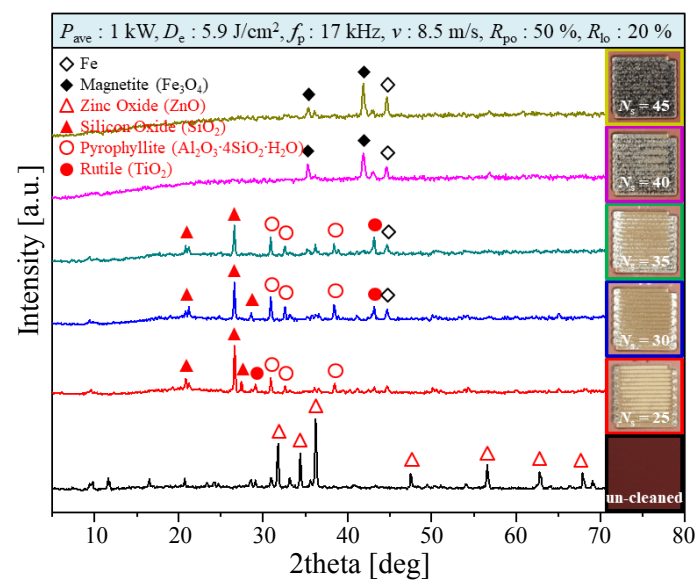

Fig. 4 XRD results of the laser cleaned surface in $D_{e}$ : $5.9 \mathrm{~J} / \mathrm{cm}^{2}$

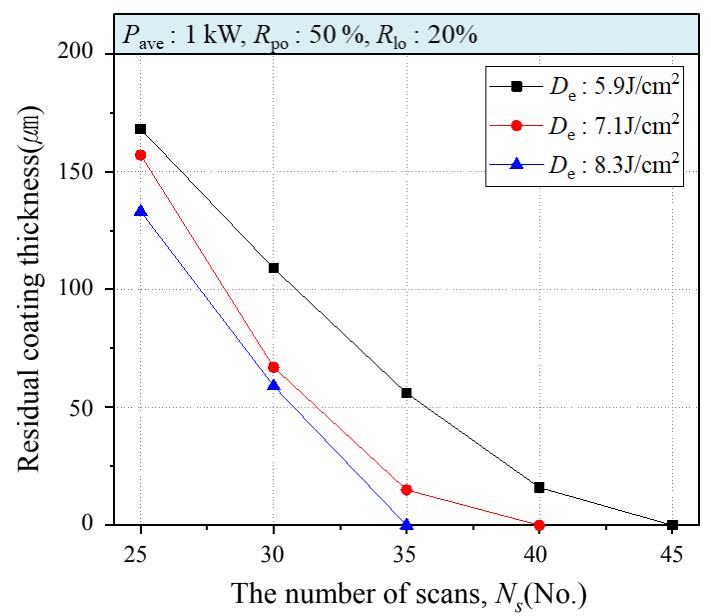

Fig. 5 Residual coating thickness distribution with the number of scans and energy density 


\subsection{Effect of average power and beam mode}

Lasers with an average power of $1 \mathrm{~kW}$ and $200 \mathrm{~W}$ were used in laser cleaning experiments that investigated the effect of average power, and the detailed test conditions are listed in Table 3. In these experiments, the beam modes of the $1 \mathrm{~kW}$ laser and $200 \mathrm{~W}$ laser were respectively set to multi mode and single mode, and the schematic of the beam profile of each laser is shown in Fig. 6. The multi mode laser exhibits a top-hat profile with a large spot diameter and uniform distribution of power density across the entire beam, while the single mode laser shows a Gaussian profile with a small spot diameter and maximum power density at the center of the beam. As such, lasers with different average power and beam mode were used to compare and analyze the cleaning effect.

Fig. 7 displays the images of the surface and cross-section of the laser cleaned area at each average power and number of scans. Coatings on the base metal were fully removed after 40 scans using the $1 \mathrm{~kW}$ laser and 16 scans using the $200 \mathrm{~W}$ laser. The reduction in cleaning effect in the former case despite the use of a laser with a higher average power is attributed to the difference in power density. Calculations on the power density $\left(D_{\mathrm{p}}\right.$, $\mathrm{W} / \mathrm{cm}^{2}$ ) affecting the removal process of each laser show that the $1 \mathrm{~kW}$ and $200 \mathrm{~W}$ lasers have a power density of $57.8 \times 10^{6}$ and $129.8 \times 10^{6}$, respectively. Hence, the performance of coating layer removal can be enhanced by using the single mode laser with a highly

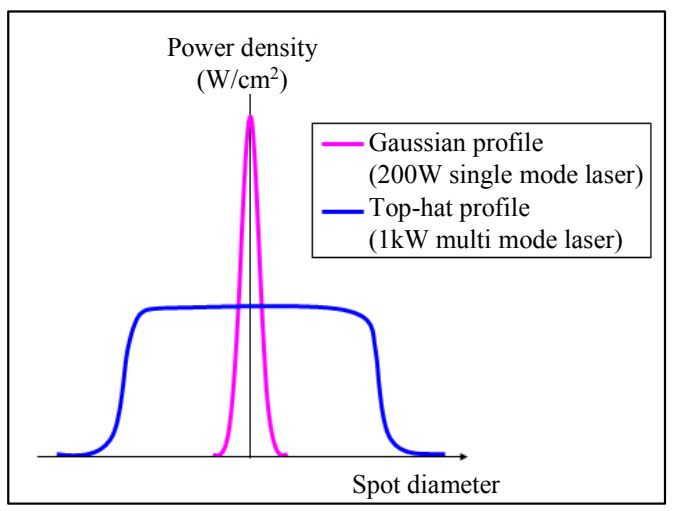

Fig. 6 Power density distribution of the gaussian and top-hat profile concentrated energy owing to the high intensity of the laser heat source penetrating into the material. In contrast, using the multi mode laser beam with a top-hat distribution resulted in an increase in the number of scans for coating layer removal due to its relatively lower power density.

\subsection{Comparison of surface characteristics}

The processing parameters of laser cleaning also have a significant effect on the surface characteristics of the cleaned area. In this section, the effect of pulse duration time $\left(\tau_{p}\right)$ and beam mode was analyzed. The laser pulse width refers to the time over which the beam irradiates from the pulse laser. As shown in the images of the laser cleaned surface at each average power (Fig. 7), using the $1 \mathrm{~kW}$ laser led to a discoloration that was caused by thermal diffusion from a relatively longer laser irradiation time resulting in thermal effects on the base metal underneath the coating layers.

In addition, the surface roughness profile of the cleaned surface at different beam modes is shown in Fig. 8. As shown in Fig. 8(a), owing to the consistent energy distribution and relatively large beam size, the laser beam with a top-hat profile leads to a smooth surface mor-

\begin{tabular}{|c|c|c|c|c|c|}
\hline$N_{\mathrm{s}}$ & Surface & $\begin{array}{l}\text { Cross- } \\
\text { section }\end{array}$ & $N_{\mathrm{s}}$ & Surface & $\begin{array}{l}\text { Cross- } \\
\text { section }\end{array}$ \\
\hline $\begin{array}{l}\text { Un- } \\
\text { clean } \\
\text { ed }\end{array}$ & & & $\begin{array}{l}\text { Un- } \\
\text { clean } \\
\text { ed }\end{array}$ & & \\
\hline 30 & & nom & 8 & & \\
\hline 35 & & & 12 & & 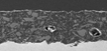 \\
\hline 40 & & & 16 & & \\
\hline & \multicolumn{2}{|c|}{ (a) $P_{\mathrm{ave}}=1 \mathrm{~kW}$} & \multicolumn{3}{|c|}{ (b) $P_{\text {ave }}=200 \mathrm{~W}$} \\
\hline
\end{tabular}

Fig. 7 Surface and cross-section of the laser cleaned surface with the average power

Table 3 Laser radiation conditions with average power

\begin{tabular}{|c|c|c|c|c|c|c|c|c|c|c|}
\hline $\begin{array}{c}\text { Parameter } \\
P_{\text {ave }}(\mathrm{W})\end{array}$ & $\begin{array}{c}\text { Beam } \\
\text { mode }\end{array}$ & $D_{\mathrm{p}}\left(\mathrm{W} / \mathrm{cm}^{2}\right)$ & $D_{\mathrm{e}}\left(\mathrm{J} / \mathrm{cm}^{2}\right)$ & $E_{\mathrm{p}}(\mathrm{mJ})$ & $f_{\mathrm{p}}(\mathrm{kHz})$ & $v(\mathrm{~m} / \mathrm{s})$ & $R_{\mathrm{po}}(\%)$ & $R_{\mathrm{lo}}(\%)$ & $\tau_{p}(\mathrm{~ns})$ & $\begin{array}{c}\text { Focal spot } \\
\text { diameter }\end{array}$ \\
\hline 1,000 & $\begin{array}{c}\text { Multi } \\
\text { mode }\end{array}$ & $57.8 \times 10^{6}$ & 8.3 & 83.3 & 12 & 6 & 50 & 20 & 100 & $1.2 \times 1.2 \mathrm{~mm}$ \\
\hline 200 & $\begin{array}{c}\text { Single } \\
\text { mode }\end{array}$ & $129.8 \times 10^{6}$ & 7.8 & 1 & 200 & 10 & 60 & 20 & 60 & $128 \mu \mathrm{m}$ \\
\hline
\end{tabular}




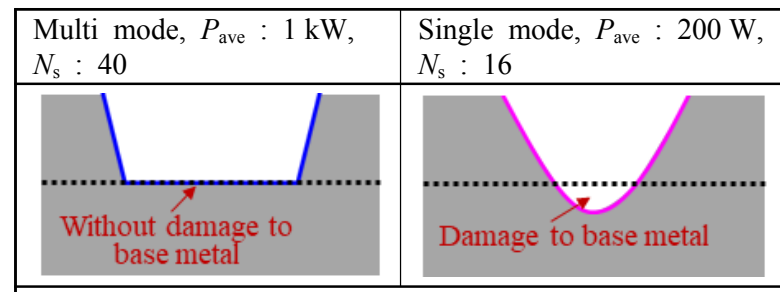

(a) Schematic of the effect of beam mode to the base metal

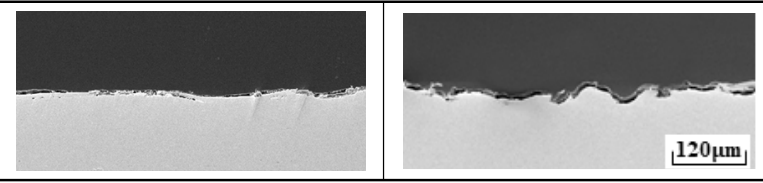

(b) Cross-section

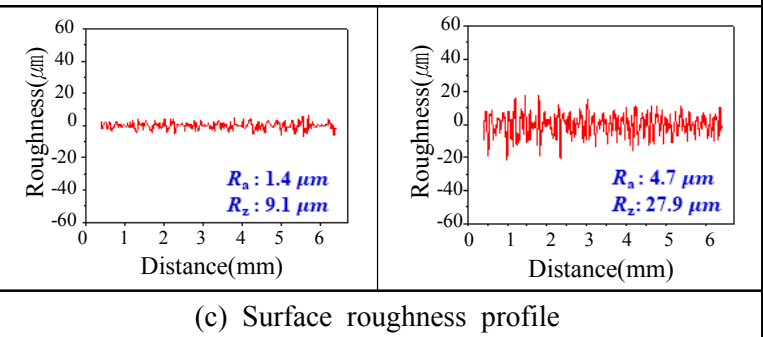

Fig. 8 Comparison of surface roughness according to beam mode

phology without damaging the base metal. On the other hand, due to the high energy intensity concentrated at the center, the laser beam with a Gaussian profile compared to that with a top-hat profile possesses a stronger intensity to penetrate into the base metal and thereby leaves a concave shape extending into the interior of the cleaned surface. Fig. 8(b) displays the images of the cross-section after removal of the coating layers. Upon comparing the two images, it can be visually confirmed that the surface of the specimen cleaned with the single mode laser is relatively rougher. The surface roughness measurements shown in Fig. 8(c) also revealed that using the laser beam with a Gaussian profile led to a three-times increase in the surface roughness.

In conclusion, laser cleaning is an environmentally friendly alternative to traditional techniques for the removal of not only coated layers of paint on ships but also various surface contaminants such as oil, grease, and oxide layers on steel surfaces, and the technology is considered as the most promising method that provides a comprehensive solution for overcoming problems such as artificial control of the surface roughness.

\section{Conclusion}

In this study, a high-power laser with an average power of $1 \mathrm{~kW}$ was used in cleaning experiments for environmentally friendly removal of multi-layer coatings applied to steel surfaces in shipbuilding, and the following conclusions were drawn.
1) Cleaning experiments under different energy density settings revealed that complete removal of multi-layer coatings was achieved with relatively smaller number of scans at a higher energy density, since the amount of laser energy irradiating on the material increased and made it easier to raise the temperature of the surface to the boiling point of the paint.

2) Analysis on the effect of average power and beam mode during laser cleaning demonstrated that a single mode laser with an average power of $200 \mathrm{~W}$ and a high power density has a strong intensity for deep penetration into the material and showed superior cleaning performance to that of the multi mode laser.

3) In addition, it was confirmed that the pulse width of a laser has a significant effect on the heat input into the material and that the laser beam mode can minimize damage to the base metal or create an artificial surface roughness.

4) Based on these research results on laser cleaning, it is believed that eco-friendly laser cleaning technology will show increased applicability in the shipbuilding and marine industry.

\section{Acknowledgments}

This work was supported by the Korea Institute for Advancement of Technology (KIAT) grant funded by the Korea Government (MOTIE) (P0008763, The Competency Development Program for Industry Specialist)

ORCID: Jong-Do Kim: http://orcid.org/0000-0002-2125-0426

ORCID: Ji-Eon Kim: http://orcid.org/0000-0003-0445-5638

ORCID: Moo-Keun Song: http://orcid.org/0000-0001-6799-1302

ORCID: Jong-Myoung Lee: http://orcid.org/0000-0001-8638-5826

\section{References}

1. H. D. Lee, Master's Degree Dissertation, Anti-corrosion properties of nanocomposite paints, University of Ulsan, Korea (2010) 1-118.

2. M. K. A. A. Razab, A. M. Noor, M. S. Jaafar, N. H. Abdullah, F. M. Suhaimi, M. Mohamed and N. Adam, A review of incorporating Nd:YAG laser cleaing principal in automotive industry, J. Radiat. Res. Appl. Sci. 11 (4) (2018) 393-402.

https://doi.org/10.1016/j.jrras.2018.08.002

3. T. Shi, C. Wang, G. Mi and F. Yan, A study of microstructure and mechanical properties of aluminum alloy using laser cleaning, J. Manuf. Proc. 42 (2019) 60-66. https://doi.org/10.1016/j.jmapro.2019.04.015

4. J. E. Kim, M. K. Song, J. M. Lee, J. H. Hyun and J. D. Kim, A Study on the Effect of Overlap Rate on Laser Beam Cleaning Characteristics while Cleaning Paint Using a Low Power Pulsed Laser( I ), J. Weld. Join. 37 
(5) (2019) 435-440.

https://doi.org/10.5781/JWJ.2019.37.5.1

5. J. E. Kim, M. K. Song, J. M. Lee, J. H. Hyun and J. D. Kim, A Study on the Effect of Overlap Rate on Laser Beam Cleaning Characteristics while Cleaning Paint Using a Low Power Pulsed Laser( П ), J. Weld. Join. 37 (5) (2019) 441-447. https://doi.org/10.5781/JWJ.2019.37.5.2

6. Jong-Do Kim, Ji-Eon Kim, Moo-Keun Song, Jong-Myoung Lee and Myoung-Soo Han, A Study on Laser Cleaning Efficiency of Epoxy Paint According to Process Parameters( I ), Trans. Korean Soc. Mech. Eng. A, 44 (3) (2020) 199-205.

https://doi.org/10.3795/KSME-A.2020.44.3.199

7. J. D. Kim, J. E. Kim, M. K. Song, J. M. Lee and M. S. Han, A Study on Laser Cleaning Efficiency of Epoxy Paint According to Process Parameters( $\Pi)$, Trans. Korean Soc. Mech. Eng. A, 44 (3) (2020) 207-212. https://doi.org/10.3795/KSME-A.2020.44.3.207

8. M. Kumar, P. Bhargava, A. K. Biswas, S. Sahu, V. Mandloi, M. O. Ittoop, B. Q. Khattak, M. K. Tiwari and L. M. Kukreja, Epoxy-paint stripping using TEA $\mathrm{CO}_{2}$ laser:Determination of threshold fluence and the process parameters, Opt. Laser Technol. 46 (2013) 29-36. https://doi.org/10.1016/j.optlastec.2012.04.021

9. D. M. D’Addona, S. Genna, A. Giordano, C. Leone, D. Matarazzo and L. Nele, Laser Ablation of Primer During the Welding Process of Iron Plate for Shipbuilding Industry, Proc. CIRP, 33 (2015) 464-469. https://doi.org/10.1016/j.procir.2015.06.055
10. J. E. Kim, M. S. Han and J. D. Kim, Removal characteristics of shop-primer paint by laser energy density in Q-switching fiber laser cleaning, Modern Phy. Lett. B, 34 (7) (2020) 2040042. https://doi.org/10.1142/S0217984920400424

11. S. Marimuthu, A. Mhich, I. S. Molchan, D. Whitehead, Z. B. Wang, P. Mativenga, L. Li, Z. Liu, C. GraftonReed, S. Cheetham and S. Dilworth, Numerical simulation of excimer laser cleaning of film and particle contaminants, J. Heat Transf. 135 (12) (2013) 121301. https://doi.org/10.1115/1.4024836

12. S. Marimuthu, A.M. Kamara, D. Whitehead, P. Mativenga and L. Li, Laser removal of TiN coatings from WC micro-tools and in-process monitoring, Opt. Laser Technol. 42 (8) (2010) 1233-1239.

https://doi.org/10.1016/j.optlastec.2010.03.016

13. Md. Shamsujjoha, S. R. Agnew, M. A. Melia, J. R. Brooks, T. J. Tyler and J. M. Fitz-Gerald, Effects of laser ablation coating removal (LACR) on a steel substrate: Part1, Surf. Coat. Technol. 281 (2015) 193-205. https://doi.org/10.1016/j.surfcoat.2015.01.071

14. Md. Shamsujjoha, S. R. Agnew, M. A. Melia, J. R. Brooks, T. J. Tyler and J. M. Fitz-Gerald, Effects of laser ablation coating removal (LACR) on a steel substrate:Part2, Surf. Coat. Technol. 281 (2015) 206-214. https://doi.org/10.1016/j.surfcoat.2015.02.034

15. M. J. J. Schmidt, L. Li and J. T. Spencer, An investigation into the feasibility and characteristics of using a $2.5 \mathrm{~kW}$ high power diode laser for paint stripping, $J$. Mater. Process. Technol. 138 (1-3) (2003) 109-115. https://doi.org/10.1016/S0924-0136(03)00057-8 